Article

\title{
Dynamics of a Dispersion-Managed Passively Mode-Locked Er-Doped Fiber Laser Using Single Wall Carbon Nanotubes
}

\author{
Norihiko Nishizawa ${ }^{1, *}$, Lei Jin ${ }^{1}$, Hiromichi Kataura ${ }^{2}$ and Youichi Sakakibara ${ }^{2}$ \\ 1 Department of Quantum Engineering, Nagoya University, Furo-cho, Chikusa-ku, \\ Nagoya 464-8603, Japan; E-Mail: kin@ nuee.nagoya-u.ac.jp \\ 2 National Institute of Advanced Industrial Science and Technology (AIST), \\ Tsukuba 305-8565, Japan; E-Mails: h-kataura@aist.go.jp (H.K.); yo-sakakibara@aist.go.jp (Y.S.) \\ * Author to whom correspondence should be addressed; E-Mail: nishizawa@ nuee.nagoya-u.ac.jp; \\ Tel.: +81-52-789-3164; Fax: +81-52-789-4437.
}

Received: 15 June 2015 / Accepted: 10 July 2015 / Published: 21 July 2015

\begin{abstract}
We investigated the dynamics of a dispersion-managed, passively mode-locked, ultrashort-pulse, Er-doped fiber laser using a single-wall carbon nanotube (SWNT) device. A numerical model was constructed for analysis of the SWNT fiber laser. The initial process of passive mode-locking, the characteristics of the output pulse, and the dynamics inside the cavity were investigated numerically for soliton, dissipative-soliton, and stretched-pulse mode-locking conditions. The dependencies on the total dispersion and recovery time of the SWNTs were also examined. Numerical results showed similar behavior to experimental results.
\end{abstract}

Keywords: fiber optics amplifiers and oscillators; nonlinear fibers; ultrafast lasers

\section{Introduction}

Single wall carbon nanotubes (SWNTs) are attractive saturable absorbers for mode-locked fiber lasers. They have a wide operating bandwidth and fast response time. Both transmission-type and reflection-type devices can be made using SWNTs. They are also cheap and easy to make. A number of groups have been working on SWNT fiber lasers [1-11]. However, one of the problems with SWNT devices is optical damage, making it difficult to realize high-power fiber lasers using SWNT devices. High-power fiber lasers are important in applications such as laser processing, nonlinear microscopy, 
and material processing. Evanescent-type devices have been investigated to improve the damage threshold $[8,12,13]$.

We have been investigating passively mode-locked, ultrashort-pulse fiber lasers using polyimide films containing dispersed SWNTs [14,15]. Recently, we demonstrated a dispersion-managed, ultrashort-pulse fiber laser using an SWNT polyimide film. High-power operation at over $140 \mathrm{~mW}$ was achieved with dissipative-soliton mode-locking operation [16]. We also achieved a dramatic increase in power up to $285 \mathrm{~mW}$ by optimizing the cavity and using a higher-power pump laser [17]. Numerical analysis is an important approach for understanding the physical mechanisms of SWNT fiber lasers and for improving the laser performance. So far, the dynamics of general ultrashort pulse fiber lasers has been reported by a few groups [18-20]. Recently, the analysis of SWNT fiber laser near zero cavity dispersion was reported by J. Wang et al. in terms of the pulse width [21].

An ultrashort-pulse SWNT fiber laser is a practical laser source that is already playing a role as a useful pulse source in optical frequency combs, ultrahigh resolution optical coherence tomography, and nonlinear microscopy [22-24]. Especially, the performance of an optical frequency comb depends on the characteristics of the laser [25-27]. Therefore, analysis of SWNT fiber lasers is important for optical frequency combs.

In this work, we carried out numerical analysis of a dispersion-managed, passively mode-locked SWNT fiber laser. A numerical model of the laser was constructed based on an experimental setup. The gain characteristics of an erbium-doped fiber (EDF) and the saturable absorption properties of an SWNT film were experimentally observed, and the results were considered in the numerical model. The initial process of passive mode-locking, the characteristics of the output pulses, and the dynamics of the mode-locked pulse inside the cavity were investigated for several different dispersion conditions. The dependencies on net cavity dispersion, recovery time, and modulation depth of the SWNTs were also examined.

\section{Methods}

\subsection{Setup of Ultrashort Pulse Fiber Laser with SWNT Film}

Figure 1 shows the configuration of the passively mode-locked, Er-doped fiber laser using an SWNT polyimide film. The cavity configuration used in Reference [16] was assumed in the numerical model. A $1.2 \mathrm{~m}$ of high-concentration EDF with positive dispersion properties (LIEKKI 110/4) was assumed as the gain medium. The second-order dispersion was $+15 \mathrm{ps}^{2} / \mathrm{km}$, and the mode-field diameter was $6.5 \mu \mathrm{m}$. The EDF was pumped by a high-power laser diode whose wavelength was $980 \mathrm{~nm}$. The output of the EDF was spliced with an isolator and was then connected with an output coupler. A variable coupler was used as the output coupler. A large part of the oscillating pulse was coupled out as the output signal, and a small part was introduced back into the ring cavity. An inline-type wavelength filter with a raised cosine bandpass function was used for constructing the laser. A normal-dispersion fiber (NDF) was used before a wavelength division multiplexed (WDM) coupler for dispersion management. The NDF had large normal dispersion properties, with $\beta_{2}=+162 \mathrm{ps}^{2} / \mathrm{km}$. The mode-field diameter was $3.0 \mu \mathrm{m}$. The length of the NDF was varied to control 
the magnitude of net cavity dispersion, $D$ T. For simplicity, the polarization state of the circulating beam was assumed to be in a stable, linearly polarized condition.

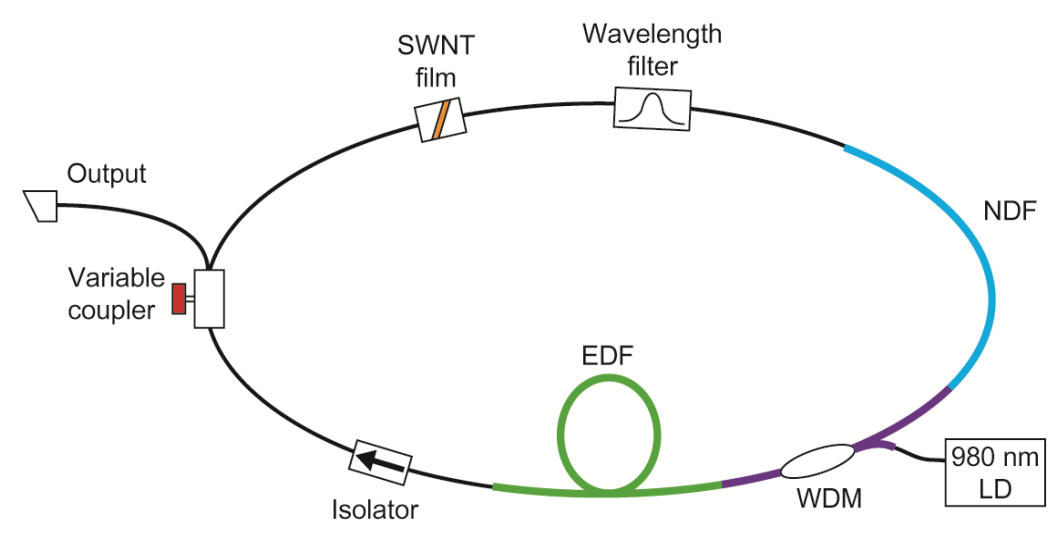

Figure 1. Configuration of passively mode-locked, Er-doped, ultrashort-pulse fiber laser with SWNT polyimide film. WDM, wavelength-division-multiplexed coupler; EDF, Er-doped fiber; NDF, normal-dispersion fiber.

As the SWNT device, in our work, we have been using a polyimide film containing dispersed SWNTs [3]. After purification, several milligrams of the SWNTs were dispersed in N-methyl-2pyrrolidone solvent under very strong ultrasonication. Then, the solution was ultracentrifuged and the supernatant was mixed with a varnish of block-copolymerized polyimide. The mixed SWNTpolyimide varnish was coated onto flat glass substrates and cured at 90 degree for $1 \mathrm{~h}$ and 180 degree for $1 \mathrm{~h}$. A self-standing film with a thickness of $25 \mu \mathrm{m}$ and an area of $2 \mathrm{~mm} \times 2 \mathrm{~mm}$ was prepared. Figure 2 shows the observed absorption spectra and saturable absorption properties of the SWNTs. A $680 \mathrm{fs}$ ultrashort pulse Er-doped fiber laser with a repetition rate of $50 \mathrm{MHz}$ and a center wavelength of $1.55 \mu \mathrm{m}$ was used for the measurement.

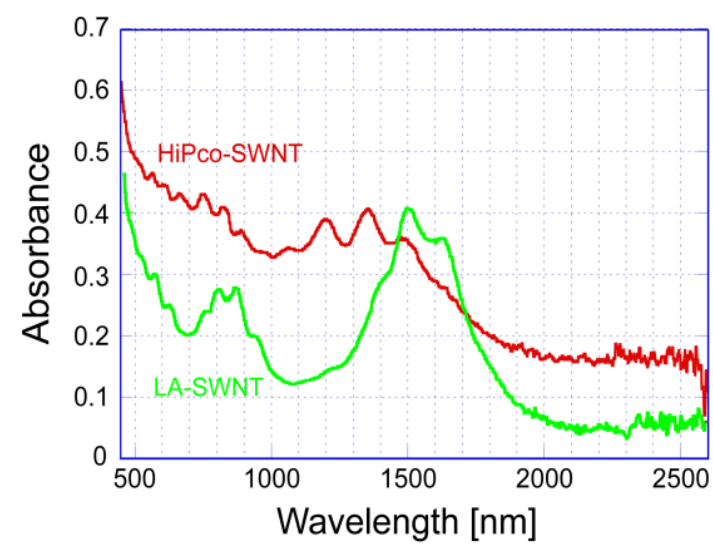

(a)

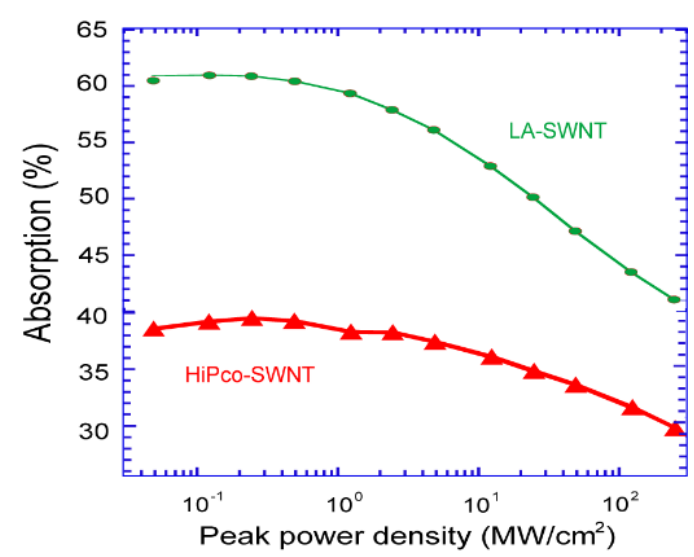

(b)

Figure 2. (a) Absorption spectra and (b) saturable absorption properties of SWNT polyimide film used in this work.

So far, we have been using SWNTs synthesized by the high-pressure CO (HiPco) method and the laser ablation (LA) method. With the HiPco method, the synthesized SWNTs showed a broad absorption spectra and a modulation depth of $\sim 10 \%$. On the other hand, with the LA method, SWNTs 
with a diameter of $1.2 \mathrm{~nm}$ were selectively synthesized, and they showed an intense absorption peak in the wavelength region around $1.55 \mu \mathrm{m}$. As shown in Figure $2 \mathrm{~b}$, the linear absorption was $61 \%$ and the $10 \%$ saturation power density was $6 \mathrm{MW} / \mathrm{cm}^{2}$. A large modulation depth of $\sim 20 \%$ was obtained for an irradiation power of $5 \mathrm{~mW}$. Owing to the low saturation power density and large modulation depth, the SWNTs work as an effective mode-locker. In this work, we assumed SWNTs synthesized by the LA method as the saturable absorber.

\subsection{Numerical Model}

In the numerical model, we used the extended nonlinear Schrödinger equation $[28,29]$ for analyzing the SWNT fiber laser:

$$
\frac{\partial A}{\partial z}+\frac{i}{2} \beta_{2} \frac{\partial^{2} A}{\partial T^{2}}-\frac{1}{6} \beta_{3} \frac{\partial^{3} A}{\partial T^{3}}+\frac{(\alpha(A, T)-g(A, T))}{2} A=i \gamma\left[|A|^{2} A+\frac{i}{\omega_{0}} \frac{\partial}{\partial T}\left(|A|^{2} A\right)-T_{R} A \frac{\partial|A|^{2}}{\partial T}\right]
$$

where $A=A(z, T)$ represents the complex electric field envelope, $z$ is distance, and $T=t-\beta_{1 z}$. The symbols $\beta_{1}, \beta_{2}$, and $\beta_{3}$ represent the magnitudes of first-, second-, and third-order dispersions. The symbols $\alpha(A, T)$ and $g(A, T)$ correspond to the optical loss and gain, respectively. The observed gain properties of the EDF were considered, and the parameters were determined to fit the experimental results. The gain saturation and the gain spectrum were considered. In this work, the gain saturation occurred for the average power, and it was given by

$$
g=\frac{g_{0}}{1+P_{a v} / P_{s g}}
$$

where $P_{a v}$ is the average power, $P_{s g}$ is the saturation power, and $g_{0}$ is the small signal gain, respectively. For the SWNT device, the observed saturable absorption properties shown in Figure 2 were fitted by the following equation

$$
\alpha=\alpha_{0}+\alpha_{m}\left(1-\frac{1}{1+P / P_{s a}}\right)
$$

where $\alpha_{0}$ is the linear loss, $\alpha_{m}$ is the saturable loss (modulation depth), $P$ is the peak power, and $P_{s a}$ is the saturation power of SWNT film. The recovery time of the SWNT device was assumed to be instantaneous, except for the case described in Section 3.5.

The right-hand side represents the nonlinear effects. The individual terms correspond to self-phase modulation, self-steepening, and Raman scattering. The split-step Fourier method was used for the numerical simulation [28]. The step size of the calculation for pulse propagation was $1-5 \mathrm{~mm}$.

In this work, amplified spontaneous emission (ASE) noise was assumed as the initial condition. The ASE noise field was obtained using a random function. A Gaussian-like spectrum centered at $1.55 \mu \mathrm{m}$ was generated by the gain properties of the EDF and a wavelength filter. For the variable coupler, an output coupling ratio of $90 \%$ was assumed, which was the value used in the previous experimental work in Reference [16]. 


\section{Results and Discussions}

\subsection{Soliton Mode-Locking Regime $\left(D_{T}<0\right)$}

First, we examined the anomalous dispersion regime, in which $D_{\mathrm{T}}=-0.05 \mathrm{ps}^{2}$. The length of the NDF was $0.2 \mathrm{~m}$, and the repetition rate was $30.8 \mathrm{MHz}$. We achieved single-pulse soliton mode-locking operation.

Figure 3 shows the variation of the pulse duration and peak power for the initial process of passive mode-locking in this regime. The bandwidth of the wavelength filter was set to be $25 \mathrm{~nm}$ full width at half-maximum (FWHM). The variations of the spectral and temporal shapes of the circulating pulse for the initial mode-locking process are shown as movies in Media 1 and 2. ASE noise circulated around the ring cavity and was amplified at the EDF for every round-trip. At the SWNT film, the intense component suffered low absorption loss owing to the saturable absorption property. Therefore, the intense component survived and was amplified at the EDF, and above a certain threshold, the amplified pulse component suffered the soliton effect, resulting in pulse compression and pulse shaping. The temporal width decreased and the peak power and spectral width rapidly increased at around 700 rounds of circulation. Then, since this intense pulse dominated the gain of the EDF, the low-power background noise component gradually decreased. Finally, a stable $\operatorname{sech}^{2}$-shaped, single ultrashort soliton pulse with Kelly sidebands was generated. Figure 4 shows the spectral and temporal waveforms of oscillating pulses at the laser output. As the numerical results, the temporal width was $265 \mathrm{fs}$, and the spectral width was $9.3 \mathrm{~nm}$ FWHM. The average power was $6.8 \mathrm{~mW}$. In the experiments, the temporal width, spectral width, and average power were $249 \mathrm{fs}, 10.0 \mathrm{~nm}$, and $2.9 \mathrm{~mW}$, respectively, when $D_{\mathrm{T}}=-0.032 \mathrm{ps}^{2}$ and the repetition rate was $39.4 \mathrm{MHz}$ [16]. The output characteristics showed similar behavior to the experimental ones. Multiple pulse oscillation was observed when the pumping power was increased.

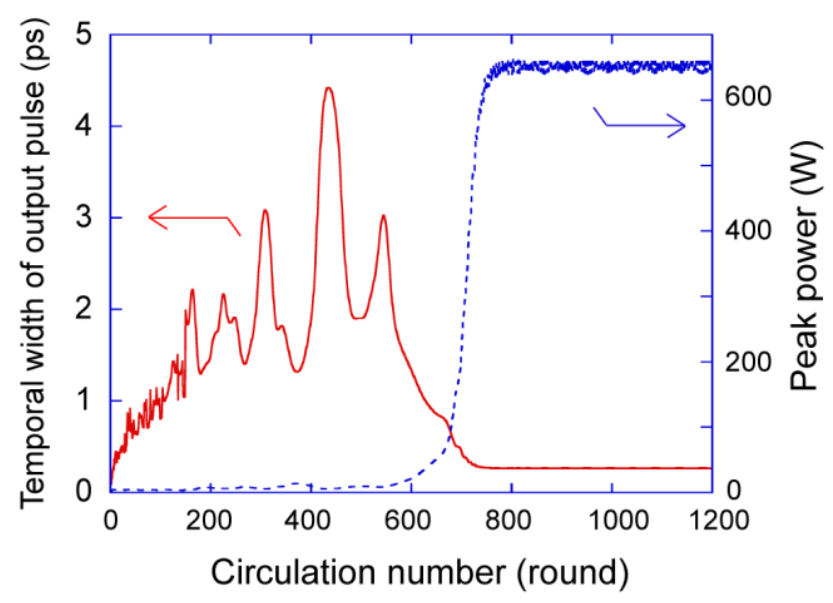

Figure 3. Variation of temporal width and peak power of output pulse for initial passive mode-locking process in soliton mode-locking regime $\left(D_{\mathrm{T}}=-0.05 \mathrm{ps}^{2}\right)$.

As shown in Figure 3, many peaks arose in the temporal width, and a stable short pulse width and high peak power were achieved due to the soliton effect. Similar behavior was almost always observed, and the same mode-locked pulse was always exactly obtained regardless of the initial ASE 
noise condition. In this analysis, a stable, clean passively mode-locked pulse was obtained after $\sim 1000$ circulations. The required number of circulations decreased as the output coupling ratio was decreased.

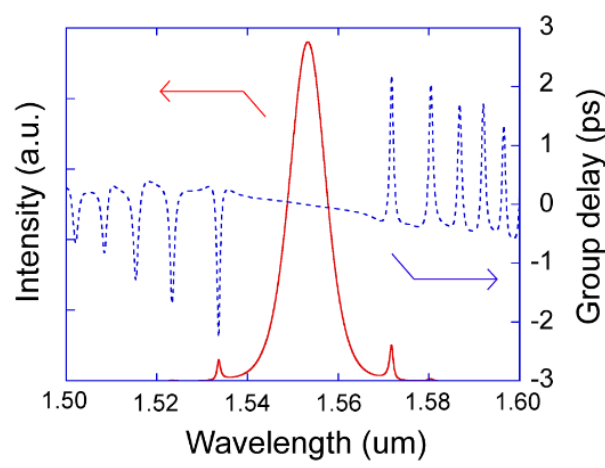

(a)

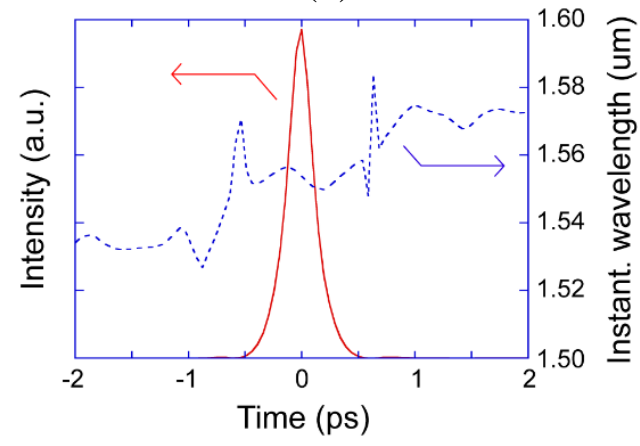

(c)

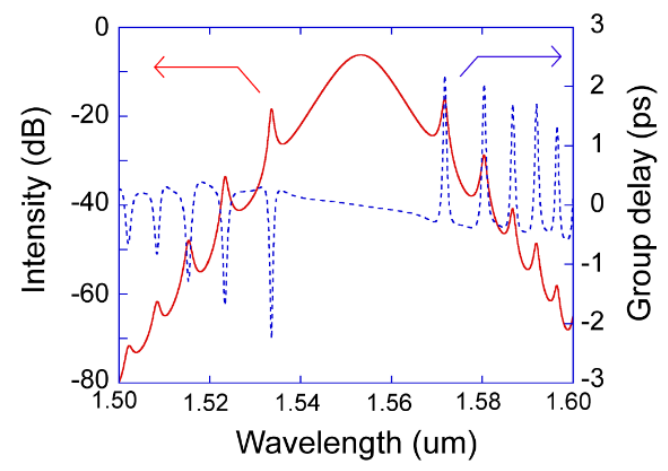

(b)

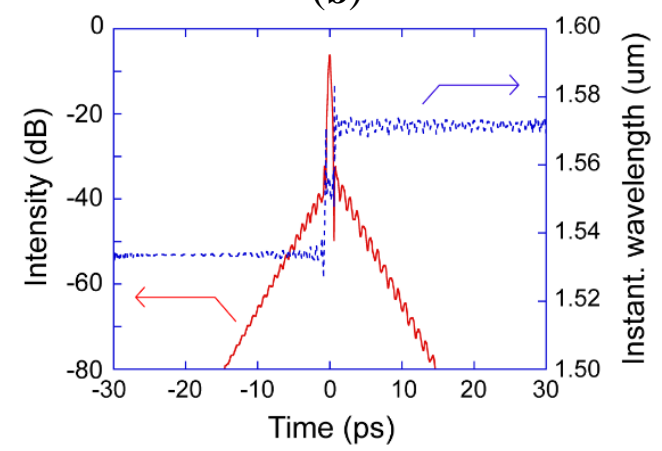

(d)

Figure 4. Characteristics of output pulse for soliton mode-locking regime without wavelength filter. (a,b) Spectral shapes on linear and log scales. (c,d) Temporal shapes on linear and log scales. Variations of spectral and temporal shapes of output pulse for initial mode-locking process are shown as movies in Media 1 and 2.

For the pulse spectra, a clear $\operatorname{sech}^{2}$-shaped spectrum with Kelly sidebands was obtained. The magnitude of chirping was small owing to the soliton effect. In the temporal shape, a wide pedestal component below $-35 \mathrm{~dB}$ was observed. From information about the instantaneous wavelength and group delay, we can see that the Kelly sideband components propagated just before and after the main pulse component, and the instantaneous wavelength of the pedestal components at the front of the main pulse was shorter than that at the center wavelength, whereas that at the back was longer.

Therefore, it is considered that the pedestal components consist of Kelly sideband components. The same correspondence was also observed experimentally by frequency resolved optical gating (FROG) in Reference [15].

Figure 5 shows the mode-locked pulse spectrum and its temporal waveform when we used a narrow-band wavelength filter to reduce the Kelly sideband components. The filter bandwidth was set to $7.8 \mathrm{~nm}$ FWHM to fit the spectral shape. We can see that the Kelly sideband components were effectively reduced by the wavelength filter, resulting in the elimination of temporal pedestal components in Figure 5. From this result, we confirmed that Kelly sideband components form the pedestal components of the output pulses in the soliton mode-locking regime. 
As experimentally shown in Reference [16], when $D_{\mathrm{T}}=-0.02 \mathrm{ps}^{2}$, the magnitude of the Kelly sidebands and the corresponding pedestal component were small compared with those when $D_{\mathrm{T}}=-0.05 \mathrm{ps}^{2}$.

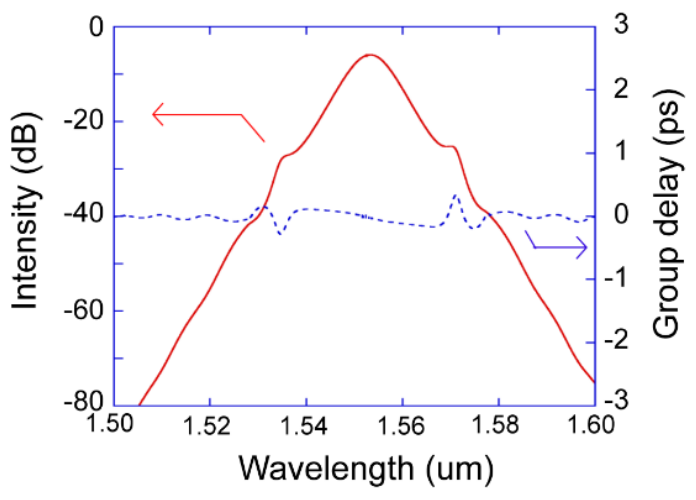

(a)

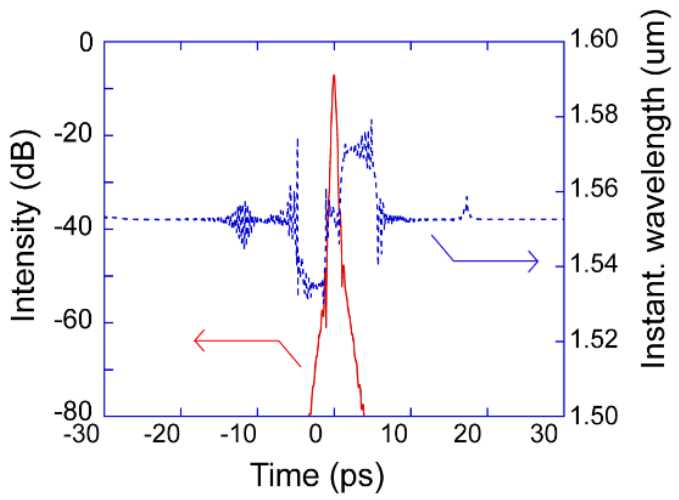

(b)

Figure 5. (a) Spectral and (b) temporal shapes of output pulses on log scale when a wavelength filter was used to remove the Kelly sideband components.

Figure 6 shows the variation of the pulse duration and peak power for a cavity round-trip in the stable soliton mode-locking regime. It is interesting to note that almost the shortest pulse was achieved at the output coupler. Since the output coupling ratio was $90 \%$, a large part of the pulse energy was picked out at the output coupler. Thus, soliton compression did not occur after the output coupler, and the pulse started to broaden due to anomalous dispersion. Then, the pulse was compressed in the NDF by normal dispersion. At the EDF, the pulse was rapidly amplified by $\sim 13 \mathrm{~dB}$ in this case. After the EDF, soliton compression occurred up until the pulse reached the output coupler owing to the high peak power. The laser output was a $\operatorname{sech}^{2}$-shaped, almost-chirp-free pulse. The spectral width was almost constant at $9.2 \pm 0.3 \mathrm{~nm}$.

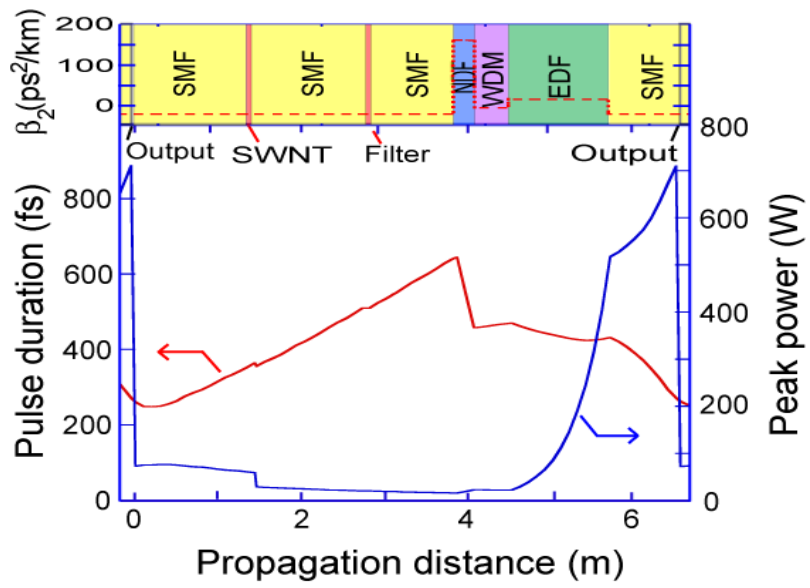

Figure 6. Dynamics of pulse propagation inside the fiber laser cavity for soliton mode-locking regime. 


\subsection{Dissipative-Soliton Mode-Locking Regime $\left(D_{T}>0\right)$}

Next, we examined the strong normal dispersion regime. The length of the NDF was $100 \mathrm{~cm}$ and $D_{\mathrm{T}}$ was $+0.08 \mathrm{ps}^{2}$. In this condition, dissipative-soliton mode-locking operation was achieved, and a high-energy picosecond pulse was generated [16,30-32]. The repetition rate was $27.4 \mathrm{MHz}$. The filter bandwidth was set to $25 \mathrm{~nm}$ FWHM.

Figure 7 shows the variation of the pulse duration and peak power for the initial process of passive mode locking. The variations of the spectral and temporal shapes of the circulating pulse are shown as movies in Media 3 and 4. Initially, both the temporal width and peak power increased as the circulation number increased. Then, the formation of a mode-locked pulse was achieved in a relatively small number of round trips $(\sim 300)$ even for a large output coupling ratio of $90 \%$. After that, the background noise level gradually decreased, and a clean, stable mode-locked dissipative soliton pulse was generated at $\sim 1100$ rounds.

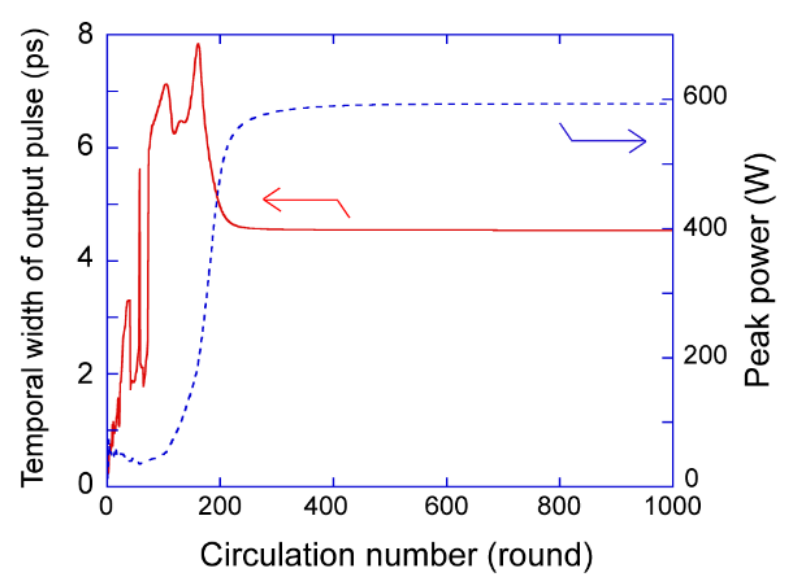

Figure 7. Variation of temporal width and peak power of output pulse for initial passive mode-locking process in dissipative-soliton mode-locking regime $\left(D_{\mathrm{T}}=+0.08 \mathrm{ps}^{2}\right)$.

Figure 8 shows the spectral and temporal shapes of the output pulses. The spectral shape had steep edges at both sides and an inclined smooth roof. The spectral width was $19.2 \mathrm{~nm}$, and the temporal width was 4.54 ps FWHM. The average power was $111 \mathrm{~mW}$. For the temporal shape, a Gaussian-like, almost linearly up-chirped picosecond pulse was generated. It is interesting to note that there was no pedestal component around the main pulse.

The shaping process of the circulating pulses can be seen in Media 3 and 4. Multiple spectral peaks appeared due to self-phase modulation (SPM) in the process of shaping the mode-locked pulse in Media 3. The variation of the temporal shape was similar to that for the soliton mode-locking regime.

Figure 9 shows the variation of the pulse duration and peak power for a cavity round-trip. The pulse had the minimum width at the input of the NDF and rapidly broadened, taking the maximum width at the output of the EDF. Then, the pulse width continuously decreased in other anomalous-dispersion fibers. Additional temporal narrowing can be seen at the SWNT film and the wavelength filter. The peak power was rapidly amplified at the EDF by $\sim 20 \mathrm{~dB}$, and the $90 \%$ component was picked off as the output. Since the peak power was suppressed, except for in EDF, the amount of induced SPM was 
well-suppressed. The high output coupling ratio is also effective in protecting against optical damage in the SWNT film. The spectral width was almost constant at $18.9 \pm 0.5 \mathrm{~nm}$.

As the bandwidth of the filter was reduced, the temporal width of the mode-locked pulse was also reduced. In the experiments, the temporal width, spectral width, and average power were $3.05 \mathrm{ps,}$ $15.6 \mathrm{~nm}$, and $105 \mathrm{~mW}$, respectively, when $D_{\mathrm{T}}=+0.082 \mathrm{ps}^{2}$ and the repetition rate was $33.8 \mathrm{MHz}$ [16]. The numerical results showed similar behavior to the experimental ones.

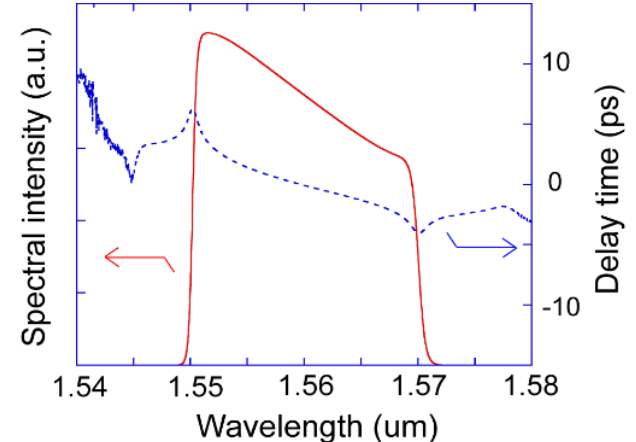

(a)

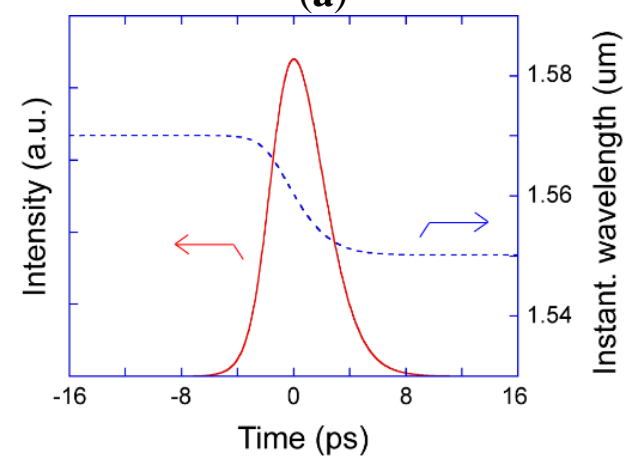

(c)

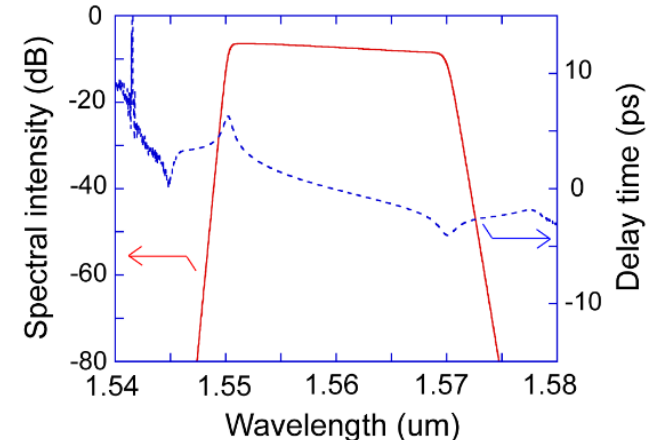

(b)

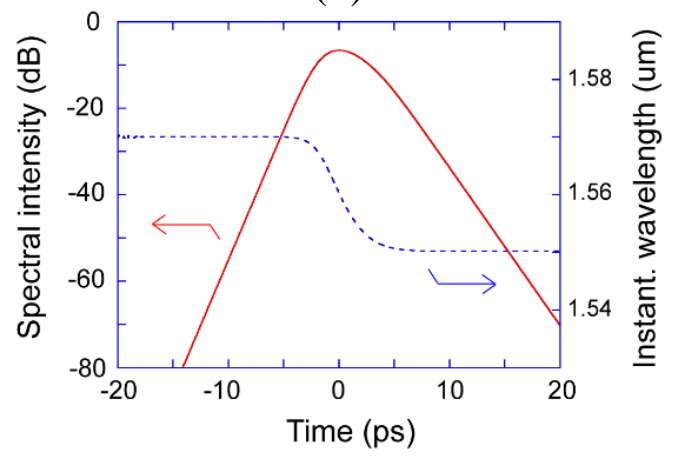

(d)

Figure 8. Characteristics of output pulse for dissipative-soliton mode-locking regime. (a,b) Spectral shapes on linear and log scales. (c,d) Temporal shapes on linear and log scales. Variations of the spectral and temporal shapes of the output pulse for the initial mode-locking process are shown as movies in Media 3 and 4.

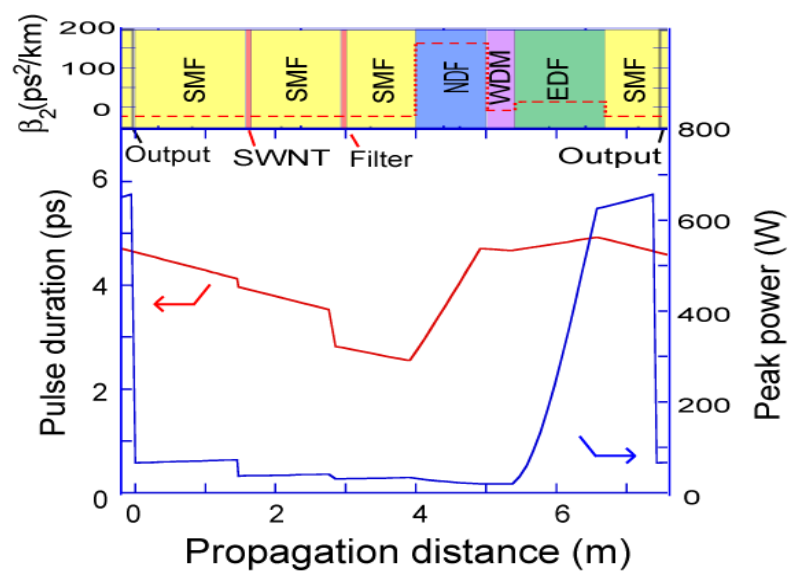

Figure 9. Dynamics of pulse propagation inside the fiber laser cavity for dissipativesoliton mode-locking regime. 


\subsection{Stretched-Pulse Mode-Locking Regime $\left(D_{T} \sim 0\right)$}

Finally, we examined the near-net-zero dispersion condition. In experiments reported in References [16,33], although multiple pulse oscillation was observed, stable single-pulse mode-locking was not achieved. In the numerical analysis, single-pulse mode-locking was also not observed for the actual properties of the SWNT film, similar to the experiment. When we assumed a deeper modulation depth twice as large as that achieved in the LA-SWNT film, single-pulse mode-locking operation was observed for the stretched-pulse mode-locking regime [33,34]. Here, we assumed $55 \mathrm{~cm}$ of NDF. The net dispersion was $D_{\mathrm{T}}=+0.007 \mathrm{ps}^{2}$. The corresponding repetition rate was $29.2 \mathrm{MHz}$. The bandwidth of the wavelength filter was set to be as wide as $100 \mathrm{~nm}$ to obtain the widest pulse spectrum.

Figure 10 shows the variation of the pulse duration and peak power for the initial process of passive mode-locking. The variations of the spectral and temporal shapes of the circulating pulse are shown as movies in Media 5 and 6. The pulse duration increased as the number of circulations increased, and it suddenly started decreasing while showing a smooth curve. At the same time, the peak power also suddenly increased, and then stable mode-locking was achieved. A small periodical modulation of $\sim 2 \%$ was observed in the peak power and pulse width.

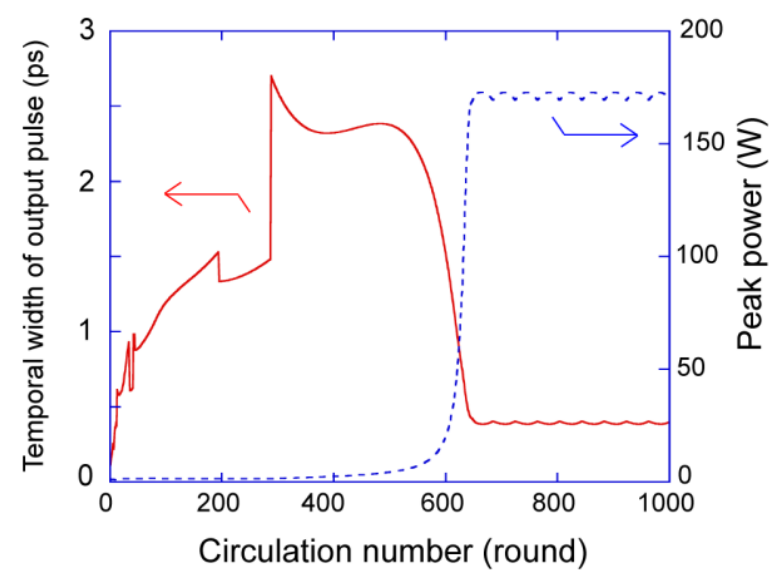

Figure 10. Variation of temporal width and peak power of output pulse for initial passive mode-locking process in dissipative soliton mode-locking condition $\left(D_{\mathrm{T}}=+0.02 \mathrm{ps}^{2}\right)$.

Figure 11 shows the spectral and temporal shapes of the mode-locked pulses at the laser output for the stretched-pulse mode-locking regime. A broad pulse spectrum with a single smooth peak was observed. As mentioned above, since the temporal shape showed almost linear up-chirping properties, the temporal shape was similar to the spectral one. An almost linear up-chirping pulse without a pedestal component was achieved. The spectral width was $22.9 \mathrm{~nm}$, and the temporal width was 390

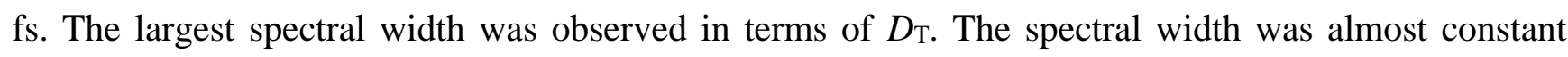
during the cavity round trip at $23.1 \pm 0.8 \mathrm{~nm}$. As the modulation depth increased, the spectral width increased.

Figure 12 shows the variation of the pulse duration and peak power for a cavity round-trip. There were two points where the temporal width was minimum: one is in the anomalous dispersion region, and the other is in the normal dispersion region. This behavior is characteristic of stretched- 
pulse mode-locking. Generally, the minimum point in normal dispersion region occurs in the EDF. For the cavity in this work, since the magnitude of normal dispersion in NDF is so large, the minimum point was observed in the NDF. The pulse showed the minimum width around the SWNT film and then started broadening. After the EDF, the pulse started narrowing, and the peak power increased. The highest peak power was achieved at the output coupler.

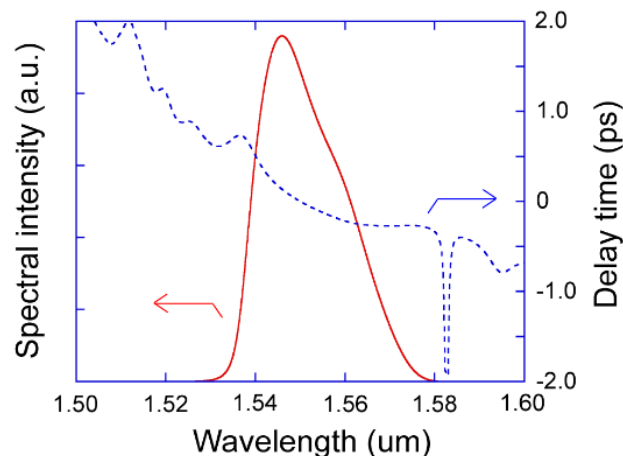

(a)

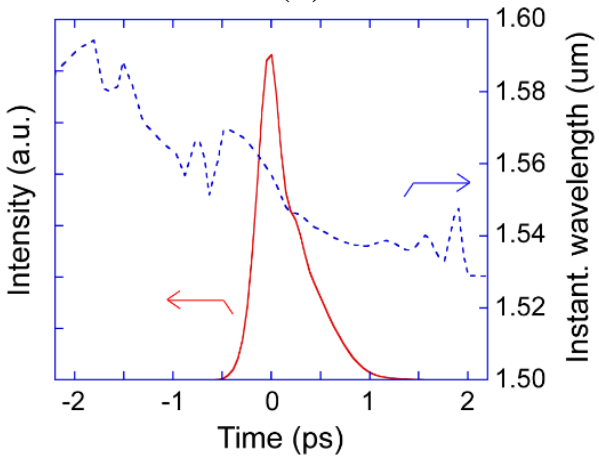

(c)

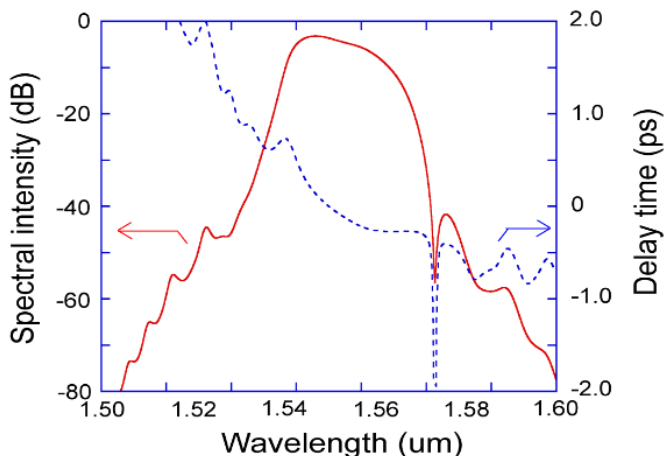

(b)

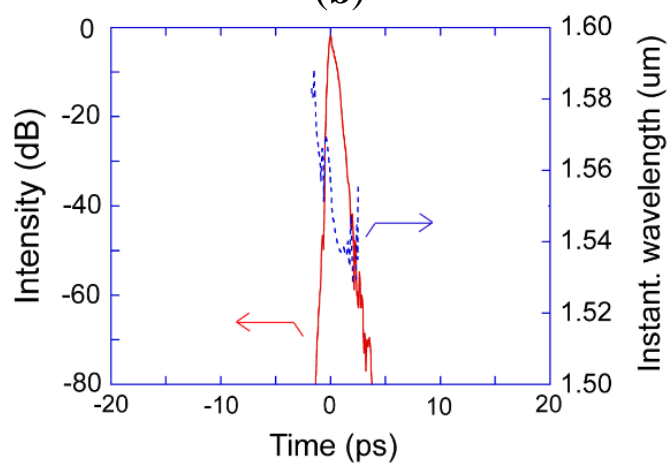

(d)

Figure 11. Characteristics of output pulse for stretched-pulse mode-locking regime. (a,b) Spectral shapes on linear and log scales. (c,d) Temporal shapes on linear and log scales. Variations of the spectral and temporal shapes of the output pulse for the initial mode-locking process are shown as movies in Media 5 and 6.

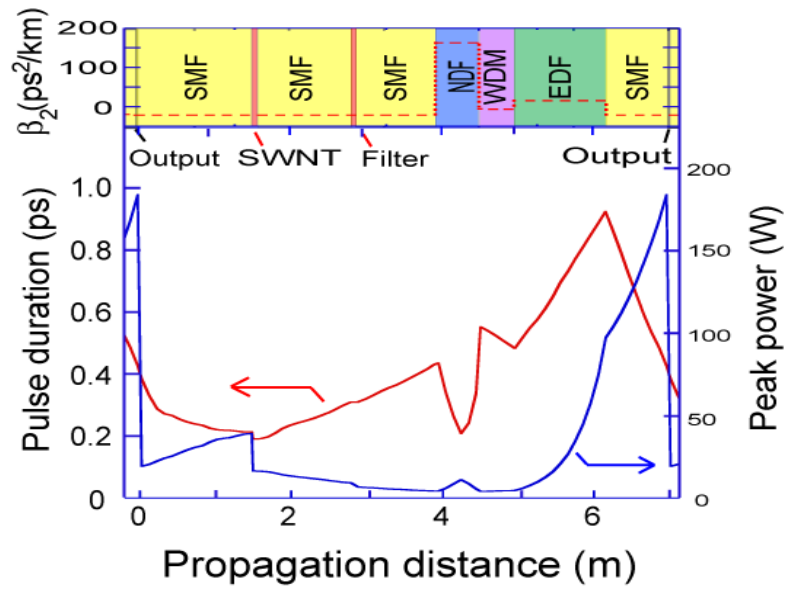

Figure 12. Dynamics of pulse propagation inside the fiber laser cavity for the stretched-pulse mode-locking regime. 


\subsection{Dependence on Net Dispersion, $D_{T}$}

With the setup shown in Figure 1, we examined the dependence of the laser performance on the net dispersion, $D_{\text {T. }}$ As in Reference [16], the length of the NDF was varied to control the amount of $D_{\text {T. }}$. Figure 13 shows the variation of the maximum average power for single-pulse mode-locking and the temporal width of the output pulse as a function of $D_{\mathrm{T}}$. For the region where $D_{\mathrm{T}} \sim 0$, the modulation depth was assumed to be twice as large as that in the actual LA-SWNT film. In agreement with the experimental results reported in Reference [16], the achievable maximum power for single-pulse oscillation and the temporal width both increased as $D_{\mathrm{T}}$ increased. We can see that, in order to achieve high pulse energy, the strong normal dispersion regime is adequate, owing to the high single-pulse oscillation limit. The maximum power was limited by the available pump power of $900 \mathrm{~mW}$ in the experiment. In a recent experiment, when we used $1.7 \mathrm{~W}$ pump power, a maximum output power of up to $285 \mathrm{~mW}$ was observed [17]. In terms of temporal width, the short pulses of 220-310 fs were obtained at $\left|D_{\mathrm{T}}\right|<0.04 \mathrm{ps}^{2}$. The shortest pulse of 220 fs was obtained for $D_{\mathrm{T}}=-0.015 \mathrm{ps}^{2}$.

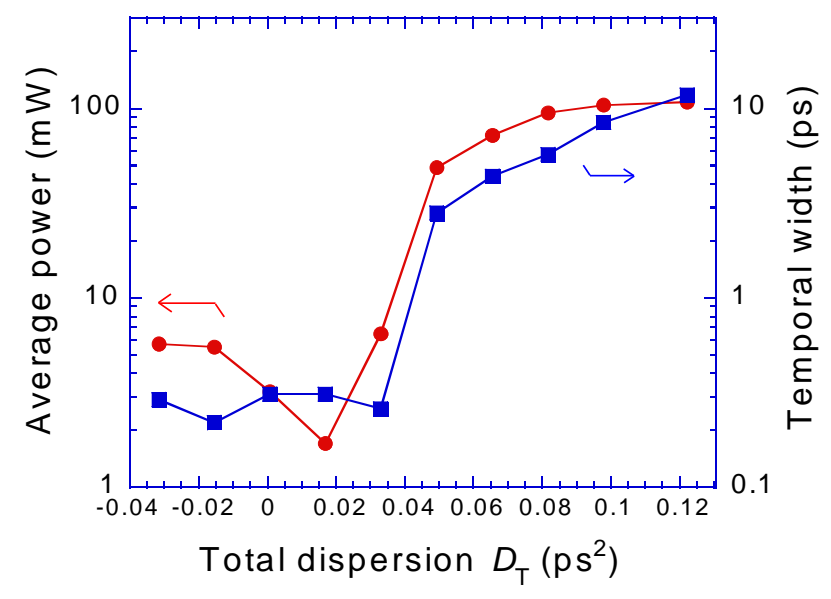

Figure 13. Variation of maximum average power and temporal width of output pulse as a function of net dispersion, $\mathrm{D}_{\mathrm{T}}$.

\subsection{Effect of Response Time and Modulation Depth of SWNT Film}

Generally, the recovery time of an SWNT film is from sub-picosecond to a few picoseconds, which is sometimes slower than the temporal width of mode-locked pulses. By the characteristic control of SWNT, such as chirality, diameter, and ratio of metallic and semiconducting SWNT, there is a possibility to control the response time of SWNT film. Thus, it is worth to investigating the effect of response time of SWNT film for fiber laser performance.

In this work, we examined the dependence of the laser performance on the response time of the SWNT film for the three mode-locking regimes that we investigated in the previous sections. Here, the recovery time up to the $90 \%$ level was varied from $10^{-13}$ to $10^{-10} \mathrm{~s}$, exponentially.

When the recovery time was less than $10^{-12} \mathrm{~s}$, clear mode-locked pulses were achieved for all three regimes. Figure 14a shows the temporal shape of the oscillating pulse and the temporal response of saturable absorption in the SWNT film for the soliton mode-locking regime. The obtained pulses were exactly the same as those for an instantaneous recovery time. The recovery time of conventional 
SWNTs is $\sim 1 \mathrm{ps,}$ which is considered fast enough not to degrade the mode-locked pulses in all three regimes. This means that the pulse formation is determined by the properties of the cavity, such as nonlinear effects, chromatic dispersion, and the characteristics of the wavelength filter.

When the recovery time of SWNT is as fast as $10^{-11} \mathrm{~s}$, the behavior depends on the value of $D$ T. For the soliton mode-locking regime, passive mode-locking was achieved, and the temporal width of the mode-locked pulse was the same as that for the instantaneous recovery time. However, if we look at the pulse carefully, there is a small noise component in the background of the mode-locked pulse, as shown in Figure 14b [35]. When the recovery time becomes slower, $10^{-10} \mathrm{~s}$, a small sub-pulse arises after the main pulse, as shown in Figure 14c. It is considered that these effects are due to the slow recovery time of the SWNT film. For the dissipative-soliton mode-locking regime, when the recovery time was slower than the temporal width of the mode-locked pulse, the temporal width was broadened as the recovery time was increased. For the stretched-pulse mode-locking regime, passive modelocking was not observed when the recovery time was larger than $10^{-11} \mathrm{~s}$.

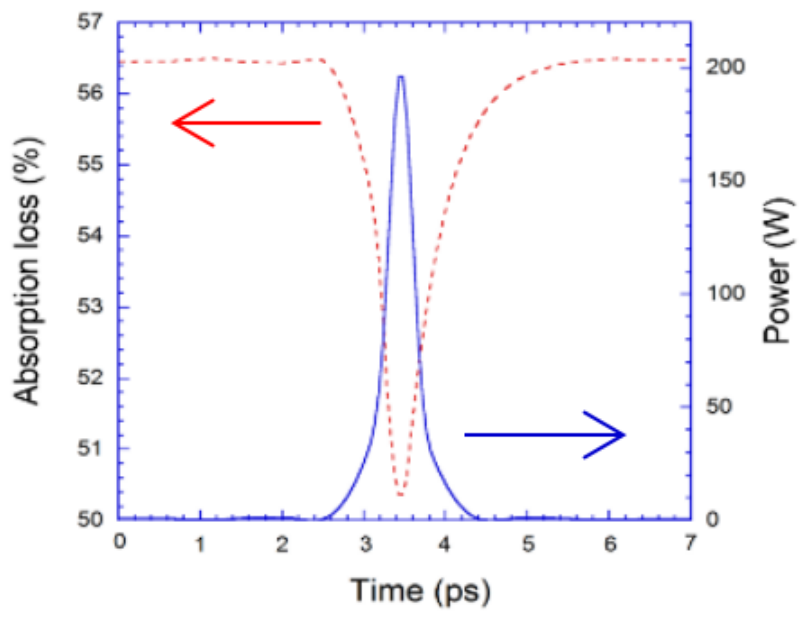

(a)

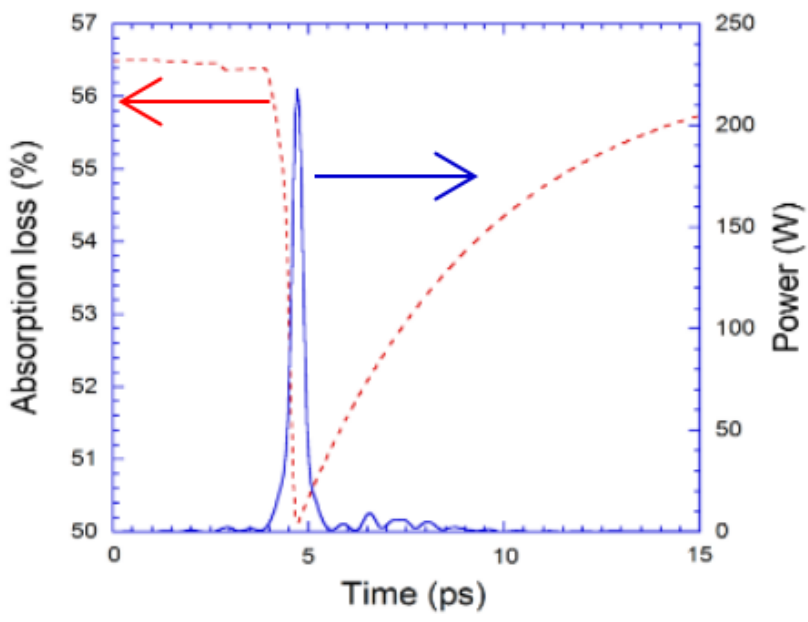

(b)

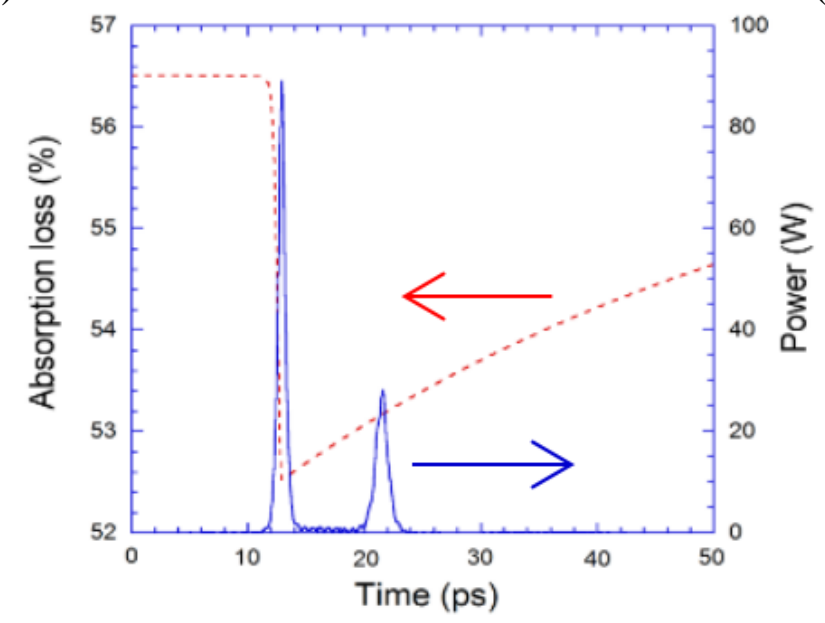

(c)

Figure 14. Temporal shapes of mode-locked pulse and variation of absorption loss at SWNT film for recovery times of (a) 0.7 ps; (b) 7 ps; and (c) 70 ps in soliton mode-locking regime. 
We also examined the effect of modulation depth for mode-locking. As mentioned previously, the large modulation depth was required to achieve stretched pulse mode-locking. For the soliton mode-locking regime, the mode-locking was achieved for the wide range of modulation depth. When the magnitude of modulation depth was smaller than $1 \%$, the background noise increased. The properties of the mode-locked pulse, however, were determined by the cavity condition, and they were independent of the modulation depth. For the dissipative soliton mode-locking regime, as the modulation depth was increased, the temporal width was decreased and the spectral width was increased.

\section{Conclusions}

In this work, we conducted numerical analysis of the dynamics of a passively mode-locked, Er-doped fiber laser using a single wall carbon nanotube (SWNT) film. A numerical model of the SWNT fiber laser was constructed based on an experimental setup. The initial passive mode-locking process, the characteristics of the output pulses, and the pulse evolution inside the cavity were investigated for soliton, dissipative-soliton, and stretched-pulse mode-locking regimes.

The dynamics of the mode-locked pulse inside the cavity strongly depended on the net dispersion, $D$ T. For the soliton mode-locking regime $\left(D_{\mathrm{T}}<0\right)$, $\operatorname{sech}^{2}$-shaped, nearly transform-limited pulses with Kelly sidebands were generated. It was confirmed that the Kelly sideband components formed the pedestal component in the temporal domain. For the dissipative-soliton mode-locking regime $\left(D_{\mathrm{T}}>0\right)$, almost linearly up-chirped pulses with a temporal width of a few picoseconds were generated. For the stretched-pulse mode-locking regime $\left(D_{\mathrm{T}} \sim 0\right)$, a larger modulation depth was required in the SWNT film to achieve single-pulse passive mode-locking. The widest pulse spectra with up-chirping properties were obtained in this regime.

We examined the dependence on the net dispersion and confirmed that a high-energy pulse could be obtained in the strong normal dispersion region. The numerical results showed similar behavior to the experimental ones.

We also examined the effect of the recovery time and modulation depth of the SWNTs, and confirmed that the mode-locked pulse was mainly formed due to the properties of the cavity, such as self-phase modulation and chromatic dispersion, and was not strictly affected by the recovery time of the SWNT in the region less than $10^{-12} \mathrm{~s}$. For a recovery time larger than $10^{-11} \mathrm{~s}$, degradation of the mode-locked pulse arose, such as increased background noise and temporal broadening.

\section{Acknowledgments}

N. Nishizawa thanks Y. Seno, Y. Ozeki, T. Konishi, and K. Itoh at Osaka University for useful discussions and cooperation.

\section{Author Contributions}

N.N. and L.J. performed experiments and simulations. H.K. and Y.S. made and characterized SWNT polyimide film. N.N. wrote the manuscript. 


\section{Conflict of Interest}

The authors declare no conflict of interest.

\section{References}

1. Set, S.Y.; Yamaguchi, H.; Tanaka, Y.; Jablonski, M.; Sakakibara, Y.; Rozhin, A.; Tokumoto, M.; Kataura, H.; Achiba, Y.; Kikuchi, K. Mode-locked fiber lasers based on a saturable absorber incorporating carbon nanotubes. In Proceedings of the Optical Fiber Communication Conference, Atlanta, GA, USA, 23-28 March 2003.

2. Yamashita, S.; Inoue, Y.; Maruyama, S.; Murakami, Y.; Yaguchi, H.; Jablonski, M.; Set, S.Y. Saturable absorbers incorporating carbon nanotubes directly synthesized onto substrates and fibers and their application to mode-locked fiber lasers. Opt. Lett. 2004, 29, 1581-1583.

3. Sakakibara, Y.; Kintaka, K.; Rozhin, A.G.; Itatani, T.; Soe, W.M.; Itatani, H.; Tokumoto, M.; Kataura, H. Optically uniform carbon nanotube-polyimide nanocomposite: Application to 165 fs mode-locked fiber laser and waveguide. In Proceedings of the 31st European Conference on Optical Communication, Glasgow, UK, 25-29 September 2005; Volume 1, p. 38.

4. Rozhin, A.G.; Sakakibara, Y.; Namiki, S.; Tokumoto, M.; Kataura, H.; Achiba, Y. Sub-200-fs pulsed erbium-doped fiber laser using a carbon nanotube-polyvinylalcohol mode locker. Appl. Phys. Lett. 2006, 88, 051118, doi:10.1063/1.2172398.

5. Nakazawa, M.; Nakahara, S.; Hirooka, T.; Yoshida, M.; Kaino, T.; Komatsu, K. Polymer saturable absorber materials in the $1.5 \mu \mathrm{m}$ band using poly-methyl-methacrylate and polystyrene with single-wall carbon nanotubes and their application to a femtosecond laser. Opt. Lett. 2006, 31, 915-917.

6. Song, Y.W.; Yamashita, S.; Goh, C.S.; Set, S.Y. Carbon nanotube mode lockers with enhanced nonlinearity via evanescent field interaction in D-shaped fibers. Opt. Lett. 2007, 32, 148-150.

7. Nicholson, J.W.; Windeler, R.S.; DiGiovanni, D.J. Optically driven deposition of single-wall carbon-nanotube saturable absorbers on optical fiber end-faces. Opt. Express 2007, 15, 9176-9183.

8. Kieu, K.; Mansuripur, M. Femtosecond laser pulse generation with a fiber taper embedded in carbon nanotube/polymer composite. Opt. Lett. 2007, 32, 2242-2244.

9. Hasan, T.; Sun, Z.; Wang, F.; Bonaccorso, F.; Tan, P.H.; Rozhin, A.G.; Ferrari, A.C. Nanotubepolymer composites for ultrafast photonics. Adv. Mater. 2009, 21, 3874-3899.

10. Sun, Z.; Hasan, T.; Ferrari, A.C. Ultrafast lasers mode-locked by nanotubes and grapheme. Phys. E 2012, 44, 1082-1091.

11. Martinez, A.; Sun, Z. Nanotube and graphene saturable absorbers for fiber lasers. Nat. Photon. 2013, 7, 842-845.

12. Song, Y.W.; Yamashita, S.; Maruyama, S. Single-walled carbon nanotubes for high-energy optical pulse formation. Appl. Phys. Lett. 2008, 92, 021115, doi:10.1063/1.2834898.

13. Im, J.H.; Choi, S.Y.; Rotermund, F.; Yeom, D. All-fiber Er-doped dissipative soliton laser based on evanescent field interaction with carbon nanotube saturable absorber. Opt. Express 2010, 18, 22141-22146. 
14. Nishizawa, N.; Seno, Y.; Sumimura, K.; Sakakibara, Y.; Itoga, E.; Kataura, H.; Itoh, K. All-polarization maintaining Er-doped ultrashort-pulse fiber laser using carbon nanotube saturable absorber. Opt. Express 2008, 16, 9429-9435.

15. Senoo, Y.; Nishizawa, N.; Sakakibara, Y.; Sumimura, K.; Itoga, E.; Kataura, H.; Itoh, K. Polarization-maintaining, high-energy, wavelength-tunable, Er-doped ultrashort pulse fiber laser using carbon-nanotube polyimide film. Opt. Express 2009, 17, 20233-20240.

16. Nishizawa, N.; Nozaki, Y.; Itoga, E.; Kataura, H.; Sakakibara, Y. Dispersion-managed, high-power, Er-doped ultrashort-pulse fiber laser using carbon-nanotube polyimide film. Opt. Express 2011, 19, 21874-21879.

17. Nozaki, Y.; Nishizawa, N.; Itoga, E.; Kataura, H.; Sakakibara, Y. Power scaling of dispersion-managed Er-doped ultrashort pulse fiber laser with single wall carton nanotubes. Opt. Lett. 2012, 37, 5079-5081.

18. Wise, F.W.; Chong, A.; Renninger, W.H. High-energy femtosecond fiber lasers based on pulse propagation at normal dispersion. Laser Photon. Rev. 2008, 2, 58-73.

19. Baumgartl, M.; Ortac, B.; Schreiber, T.; Limpert, J.; Tunnermann, A. Ultrashort pulse formation and evolution in mode-locked fiber lasers. Appl. Phys. B 2011, 104, 523-536.

20. Renninger, W.H.; Chong, A.; Wise, F.W. Pulse shaping and evolution in normal-dispersion mode-locked fiber lasers. IEEE J. Select. Top. Quantum Electron. 1999, 5, 1385-1391.

21. Wang, J.; Cai, Z.; Xu, P.; Du, G.; Wang, F.; Ruan, S.; Sun, Z.; Hasan, T. Pulse dynamics in carbon nanotube mode-locked fiber lasers near zero cavity dispersion. Opt. Express 2015, 23, 19947-19958.

22. Wu, T.H.; Kieu, K.; Peyghambarian, N.; Jones, R.J. Low noise erbium fiber fs frequency comb based on a tapered-fiber carbon nanotube design. Opt. Express 2011, 19, 5313-5318.

23. Kawagoe, H.; Ishida, S.; Aramaki, M.; Sakakibara, Y.; Omoda, E.; Kataura, H.; Nishizawa, N. Development of a high power supercontinuum source in the $1.7 \mathrm{um}$ wavelength region for highly penetrative ultrahigh-resolution optical coherence tomography. Biomed. Opt. Express 2014, 5, 932-943.

24. Freudiger, C.W.; Yang, W.; Holtom, G.R.; Peyghambarian, N.; Xie, X.S.; Kieu, K.Q. Stimulated Raman scattering microscopy with a robust fiber laser source. Nat. Photon. 2014, 8 , 153-159.

25. Newbury, N.R.; Washburn, B.R. Theory of the frequency comb output from a femtosecond fiber laser. IEEE J. Quant. Electron. 2005, 41, 1388-1402.

26. Nakajima, Y.; Inaba, H.; Hosaka, K.; Monishima, K.; Onae, A.; Yasuda, M.; Kohno, T.; Kawato, S.; Kobayashi, T.; Katsuyama, T.; et al. A multi-branch, fiber-based frequency comb with millihertz-level relative linewidths using an intra-cavity electro-optic modulator. Opt. Express 2010, 18, 1667-1676.

27. Glandorf, L.N.; Johnson, T.A.; Kobayashi, Y.; Diddams, S.A. Impact of dispersion on amplitude and frequency noise in a Yb-fiber laser comb. Opt. Lett. 2011, 36, 1578-1580.

28. Agrawal, G.P. Applications of Nonlinear Fiber Optics, 2nd ed.; Academic Press: San Diego, CA, USA, 2008. 
29. Schreiber, T.; Ortac, B.; Limpert, J.; Tunnermann, A. On the study of pulse evolution in ultra-short pulse mode-locked fiber lasers by numerical simulations. Opt. Express 2007, 15, 8252-8262.

30. Chong, A.; Buckley, J.; Renninger, W.; Wise, F. All-normal-dispersion femtosecond fiber laser. Opt. Express 2006, 14, 10095-10100.

31. Cabasse, A.; Ortac, B.; Martel, G.; Hideur, A.; Limpert, J. Dissipative solitons in a passively mode-locked Er-doped fiber with strong normal dispersion. Opt. Express 2008, 16, 19322-19329.

32. Bale, B.G.; Boscolo, S.; Turitsyn, S.K. Dissipative dispersion-managed solitons in mode-locked lasers. Opt. Lett. 2009, 34, 3286-3288.

33. Kieu, K.; Wise, F.W. Self-similar and stretched-pulse operation of Erbium-doped fiber lasers with carbon nanotubes saturable absorber. In Proceedings of the Conference on Laser Electro-Optics, Baltimore, MD, USA, 2-4 June 2009.

34. Tamura, K.; Ippen, E.P.; Haus, H.A.; Nelson, L.E. 77-fs pulse generation from a stretched-pulse mode-locked all-fiber ring laser. Opt. Lett. 1993, 18, 1080-1082.

35. Kartner, F.X.; der Au, J.A.; Keller, U. Mode-locking with slow and fast saturable absorbers-What's the difference? IEEE J. Select. Top. Quantum Electron. 1998, 4, 159-168.

(C) 2015 by the authors; licensee MDPI, Basel, Switzerland. This article is an open access article distributed under the terms and conditions of the Creative Commons Attribution license (http://creativecommons.org/licenses/by/4.0/). 